\title{
Shrinktech: A Tool for the Robustness Analysis of Timed Automata ${ }^{\star}$
}

\author{
Ocan Sankur \\ LSV, ENS Cachan \& CNRS, France \\ sankur@lsv.ens-cachan.fr
}

\begin{abstract}
We present a tool for the robustness analysis of timed automata that can check whether a given time-abstract behaviour of a timed automaton is still present when the guards are perturbed. The perturbation model we consider is shrinking, which corresponds to increasing lower bounds and decreasing upper bounds in the clock guards by parameters. The tool synthesizes these parameters for which the given behaviour is preserved in the new automaton if possible, and generates a counter-example otherwise. This can be used for 1) robustness analysis, and for 2) deriving implementations under imprecisions.
\end{abstract}

\section{Introduction}

Timed Automata and Robustness. Timed automata 3] are a well-established formal model for real-time systems. They can be used to model systems as finite automata, while using, in addition, a finite number of clocks to impose timing constraints on the transitions. Timed automata are, however, abstract models, and therefore make unrealistic assumptions on timings, such as perfect continuity of clocks, infinite-precision time measures and instantaneous reaction times. An important amount of work has been done in the timed automata literature to endow timed automata with a realistic semantics. The works [14] and [10] showed that perturbations on clocks, either imprecisions or clock drifts, and regardless of how small they are, may yield additional qualitative behaviours in some timed systems. On the other hand, assuming bounds on the reaction times can disable desired behaviours [81. These observations mean that there is a need for checking the robustness of timed automata models, that is, whether the behaviour of a given timed automaton is preserved in presence of small perturbations. Robustness is an important property of critical embedded systems [12, since it requires that the system will behave correctly when the environment's behaviour deviates slightly from the assumptions.

Clock Imprecisions and Shrinking. A prominent approach to model imprecisions in timed automata, initiated in [11, consists in introducing imprecisions in the model by syntactically enlarging all guards, that is, turning a guard $x \in[a, b]$ into $x \in[a-\Delta, b+\Delta]$ for some parameter $\Delta>0$. Model-checking algorithms on

* This work has been partly supported by project ImpRo (ANR-10-BLAN-0317). 
timed automata have been revisited in order to take into account such imprecisions (see e.g. 106]). These algorithms check whether any really new behaviour appears when timing constraints are relaxed by a small (parameterized) amount.

Recently we studied the dual notion of robustness, which consists in checking whether any behaviour is lost when the guards are shrunk, that is tightened by a small (parameterized) amount. More precisely, shrinking means converting a guard $x \in[a, b]$ into $x \in\left[a+\delta, b-\delta^{\prime}\right]$ for some $\delta, \delta^{\prime}>0$. In [15, we showed that one can decide whether all guards can be shrunk -by possibly different amounts, so that the resulting timed automaton can still time-abstract simulate the original automaton. In this case, one can also synthesize these shrinking parameters for each atomic guard. By checking shrinkability of timed automata, one ensures that the behaviour of the automaton does not depend on exact timings, or on its ability to take the transitions on the boundaries of the guards. A shrinkable timed automaton preserves all its behaviours when, for instance, task execution times are shorter than the worst-case, and waiting times are longer than the best-case. One can also detect unrealistic runs, including Zeno runs [15]. We believe that shrinkability complements the robustness approach based on guard enlargement of [1110].

Shrinkability can also be used for deriving implementations with imprecise clocks. In fact, if the guard $x \in[a, b]$ is shrunk into $x \in[a+\delta, b-\delta]$, then under imprecisions modelled by guard enlargement (as in [1]), this guard becomes $x \in[a+\delta-\Delta, b-\delta+\Delta] \subseteq[a, b]$, where the inclusion holds whenever $\Delta<\delta$. Hence, the behaviours of a shrunk timed automaton with bounded imprecisions (i.e. guard enlargement) are entirely included in the behaviours of the initial timed automaton. Further, using shrinkability, one can synthesize parameters $\delta$ for each guard, so that the resulting automaton still contains some useful timeabstract behaviour.

Related Work. Existing verification tools for timed automata may be used for non-parameterized robustness checking by modeling explicitly the imprecisions, although this increases the size of the models [2]. The semi-algorithm of HyTech was used to synthesize guard enlargement parameters in timed automata in 11. An extension of Uppaal for robustness against guard enlargement was used in 13]; this feature is no longer available in Uppaal. Note that shrinkability cannot be solved by existing model-checkers for timed automata since we are interested in parameter synthesis so as to ensure time-abstract simulation. Other similar work includes (the undecidable problem of) parameter synthesis in timed automata, where guards are written using parameters, and one tries to find the valuations for which the system satisfies some specification, e.g. [4]. This is difficult to realize due to the large number of parameters (upto millions) and the time-abstract simulation condition we consider. Robustness against large decreases in task execution times using simulation was considered in [1].

\section{Shrinkability}

Let us define shrinkability more formally. We assume that the reader is familiar with the syntax and semantics of Alur-Dill timed automata, and refer to [3] for 


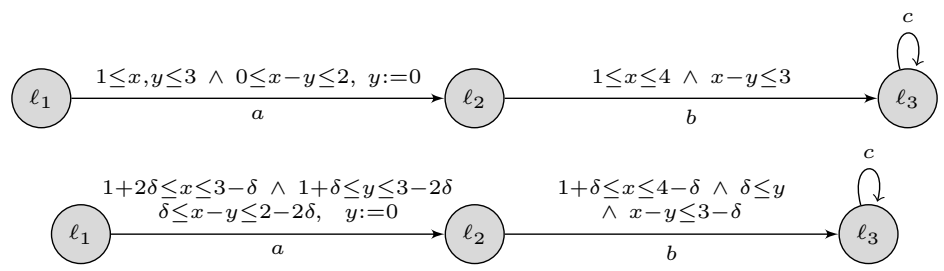

Fig. 1. A timed automaton $\mathcal{A}$ (above) and its shrinking $\mathcal{A}_{-\mathbf{k} \delta}$ (below). Timed automaton $\mathcal{A}_{-\mathbf{k} \delta}$ can time-abstract simulate $\mathcal{A}$ for all $\delta \in\left[0, \frac{1}{6}\right]([15])$.

details. We only need the following definitions. Given a finite clock set $\mathcal{C}$, an atomic guard is an expression of the form $x \leq k|x \geq k| x-y \leq k$, where $x, y \in \mathcal{C}$ and $k \in \mathbb{Z}$. A guard is a conjunction of atomic guards. The shrinking of an atomic guard $g$ by $\delta$, denoted by $\langle g\rangle_{-\delta}$ is defined as $\langle x \leq k\rangle_{-\delta}=x \leq k-\delta$, $\langle x \geq k\rangle_{-\delta}=x \geq k+\delta$, and $\langle x-y \leq k\rangle_{-\delta}=x-y \leq k-\delta$.

Note that the only variables that appear in timed automata are clocks. Discrete variables with bounded domains can be considered, but we assume these are encoded in the locations.

Let $\mathcal{A}$ be a timed automaton, and let $I$ be the vector of the atomic guards of $\mathcal{A}$. Given a vector $\boldsymbol{\delta}$ of nonnegative rational numbers indexed by $I$, we denote by $\mathcal{A}_{-\boldsymbol{\delta}}$, the automaton obtained from $\mathcal{A}$ by shrinking each atomic guard by the corresponding element of $\boldsymbol{\delta}$. We are going to write the vector $\boldsymbol{\delta}$ as $\boldsymbol{k} \delta$ for an integer vector $\boldsymbol{k}$ and rational $\delta$. This is always possible since we are interested in rational parameters. Figure 1 is an example of shrinking.

We are interested in shrinking the atomic guards of a given timed automaton by positive values, while preserving some of the behaviours. We only consider timed automata with non-strict guards; in fact, using strict guards makes little sense when one is interested in shrinking (or enlarging) the guards [10. We also assume that the edges have distinct labels, since we are interested in comparing two timed automata that have the same underlying structure. The problem is formulated as follows:

Definition 1 (Shrinkability). Given a timed automaton $\mathcal{A}$, and a finite automaton $\mathcal{F}$ such that $\mathcal{F} \sqsubseteq_{t a} \mathcal{A}$, decide whether for some $\boldsymbol{\delta}>0, \mathcal{F} \sqsubseteq_{t a} \mathcal{A}_{-\boldsymbol{\delta}}$.

In this definition, $\sqsubseteq_{\text {ta }}$ denotes time-abstract delay simulation. We say that $\mathcal{A}$ is shrinkable w.r.t. $\mathcal{F}$ if the above condition is satisfied. Thus, shrinkability requires that some behaviour $\mathcal{F}$, that is included in the initial model $\mathcal{A}$, should be still possible in the shrunk automaton. When $\mathcal{F}$ is the region graph of $\mathcal{A}[3]$, or a time-abstract bisimulation quotient [16], shrinkability implies that the shrunk automaton can time-abstractly simulate the original automaton. In this case, we say that $\mathcal{A}$ is simply shrinkable. Shrinkability w.r.t. $\mathcal{F}$ is decidable in polynomial time if $\mathcal{F}$ is part of the input; shrinkability is decidable in exponential time, if the time-abstract bisimulation graph is not given. The vector $\boldsymbol{\delta}$ can be computed in the same time complexity [15]. 


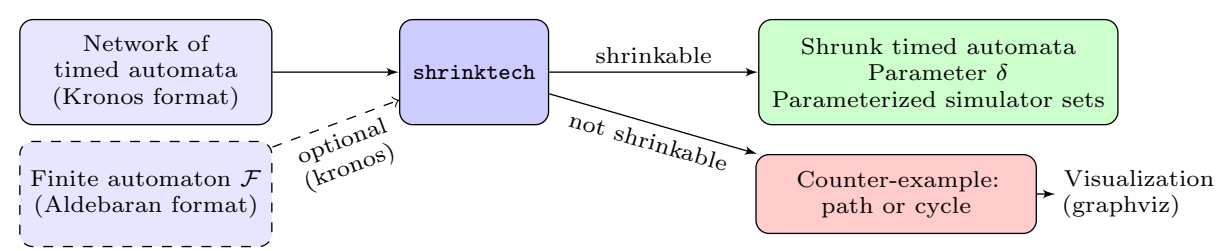

Fig. 2. Overview of shrinktech

The present tool builds on the theoretical results presented in [15. There, we show that given a finite automaton $\mathcal{F}$, the shrinking parameter of each atomic guard can be expressed as a function of the other parameters using only maximization and sum. The problem is then reduced, in polynomial time, to solving nonlinear fixpoint equations in the max-plus algebra. We gave in [15] graph-based algorithms to solve these equations.

Partial shrinkability. Although we defined shrinkability by requiring that all atomic guards should be shrunk by a positive amount, one can relax this condition and shrink only some of the guards; our algorithms are valid also in this case. In our experiments, we shrunk all the guards but equality constraints.

\section{The Tool shrinktech}

We present the tool shrinktech that analyzes the shrinkability of timed automata and synthesizes shrinking parameters. Given a network of timed automata, the tool either finds a counter-example to shrinkability, such as a path or a cycle that cannot be executed by any shrinking of the automaton, whatever the value of $\delta$ 's are, or outputs a shrinking of the timed automata that witnesses the shrinkability.

Figure 3 shows an overview of the tool. To check the shrinkability of a timed automaton, the user can either provide a finite automaton $\mathcal{F}$, or let shrinktech compute the full finite bisimilarity graph using Kronos 1 . Note that if the full bisimilarity graph is too big, one can also try to shrink with respect to a portion of it, or with respect to a randomly generated trace. This is to be compared with bounded model-checking, which is useful for detecting bugs, but also for "partially" proving the correctness of a system. The tool comes with scripts to compute the bisimilarity graph, extract some (random) portion of it, and generate random executions.

The tool shrinktech can be used for several kinds of systems modelled by timed automata. We believe it can be used mainly for two purposes:

1. Robustness analysis, to find out whether the behaviour of the system is preserved when the time bounds are disturbed (shrunk). This analysis complements the robustness checking by enlarging the guards as in [112]. This

\footnotetext{
${ }^{1}$ Kronos is a model-checker for timed automata 7] that can minimize the region graph of a timed automaton as described in [16. It is available at http://www-verimag.imag.fr/DIST-TOOLS/TEMPO/kronos/
} 
can for instance help to detect unrealistic executions such as Zeno or other convergence phenomena, but also timing anomalies in scheduling problems (see 5]).

2. Deriving implementations from timed automata. As explained above, the behaviour of a shrunk timed automaton is included in that of the initial model in presence of imprecisions. So lower and upper bounds on the delays can be "shrunk" in the implementation to guarantee that these will be respected despite imprecisions. In fact, we proved in [15] that shrinkable timed automata can be implemented in a concrete semantics with imprecise clocks and reaction times.

Implementation details and availability. The tool is implemented in $\mathrm{C}++$ and the source code has about 5Klocs. It uses the Uppaal DBM library2, and implements a parameterized extension of this data structure, introduced in [15. The input formats are (networks of) timed automata in the Kronos format, and finite automata in the Aldebaran format 3 . The tool Kronos can be plugged in the tool-chain in order to compute the finite time-abstract bisimilarity graph of a given timed automaton, to be used as the finite automaton $\mathcal{F}$. Shrinktech is open source software and is distributed under GNU General Public Licence 3.0. It is freely available at: http://www.lsv.ens-cachan.fr/Software/shrinktech

\section{Experimental Results}

We used shrinktech on several case studies found in the literature. The table 1 summarizes the results. The Lip Synchronization Protocol has been the subject of robustness analysis (by guard enlargement) before [13. This is an algorithm that synchronizes video and sound streams that arrive in different frequencies. The model is not shrinkable neither for video frames arriving in exact frequency, nor for those arriving within a bounded interval. Observe that the model is shrinkable w.r.t. a small subgraph with 501 nodes, but it is not shrinkable w.r.t. the whole graph, which has 4484 nodes. Shrinkable models include Philips Audio Retransmission protocol [9], and some asynchronous circuit models. We were able to analyze Fischer's Mutual Exclusion Protocol upto 4 agents; while for 5 agents we could only partially analyze w.r.t. a randomly generated trace. The non-shrinkability of most models is due to equality constraints. In fact, although we only shrink non-punctual guards, some behaviours may still disappear immediately, however small the shrinking parameter is.

Note that some of these models were designed at a level of abstraction where imprecisions were not taken into account. So, our results do not necessarily imply that these systems are not robust, but rather that the present models are not good for direct implementation. This is best illustrated in the Latch Circuit models, where the exact model that extensively uses equalities is not shrinkable,

2 http://people.cs.aau.dk/ adavid/UDBM/

3 This is a graph description format of the CADP tool suite, also used by Kronos. See http://www.inrialpes.fr/vasy/cadp/ 
Table 1. The column sim-graph is the number of states and the number of transitions of the finite automaton $\mathcal{F}$ w.r.t. which the shrinkability is checked. An asterisk indicates bounded shrinkability, where only a subgraph of the time-abstract bisimulation graph (given by a BFS) or a random trace was used. The tests were performed on an Intel Xeon $2.67 \mathrm{GHz}$. All models are available on the tool's website.

\begin{tabular}{|l|l|l|l|l||l|l|}
\hline Model & states & trans & clocks & sim-graph & time & shrinkable \\
\hline Lip-Sync Prot. (Exact) & 230 & 680 & 5 & $4000 / 8350^{*}($ subgraph) & 9s & No \\
\hline Lip-Sync Prot. (Interval) & 230 & 680 & 5 & $501 / 1282^{*}($ subgraph) & $9 \mathrm{~s}$ & Yes* \\
\hline Lip-Sync Prot. (Interval) & 230 & 680 & 5 & $4484 / 48049$ & $28 \mathrm{~s}$ & No \\
\hline Philips Audio Prot. & 446 & 2097 & 2 & $437 / 2734$ & $46 \mathrm{~s}$ & Yes \\
\hline Root Contention Prot. & 65 & 138 & 6 & $500 / 3455^{*}$ (subgraph) & $7 \mathrm{~s}$ & No \\
\hline Train Gate Controller & 68 & 199 & 11 & $952 / 8540$ & $34 \mathrm{~s}$ & No \\
\hline Fischer's Protocol 3 & 152 & 464 & 3 & $472 / 4321$ & $20 \mathrm{~s}$ & Yes \\
\hline Fischer's Protocol 4 & 752 & 2864 & 4 & $4382 / 65821$ & $310 \mathrm{~min}$ & Yes \\
\hline Fischer's Protocol 5 & 3552 & 16192 & 5 & $10000 / 10000^{*}$ (trace) & $42 \mathrm{~s}$ & Yes* \\
\hline And-Or Circuit & 12 & 20 & 4 & $80 / 497$ & $1.3 \mathrm{~s}$ & Yes \\
\hline Flip-Flop Circuit & 22 & 34 & 5 & $30 / 64$ & $0.9 \mathrm{~s}$ & Yes \\
\hline Latch Circuit (Interval) & 32 & 77 & 7 & $105 / 364$ & $1.6 \mathrm{~s}$ & Yes \\
\hline Latch Circuit (Exact) & 32 & 77 & 7 & $100 / 331$ & & No \\
\hline
\end{tabular}

but its relaxation to intervals is. Notice also how most of the circuit models which define bounds on stabilization times are shrinkable.

Our approach depends on the computation of the finite automaton $\mathcal{F}$, thus it is limited by the feasibility of this computation. To deal with this problem, one could consider adapting the algorithm of [16] to compute on-the-fly some bounded part of the graph. To be able to treat even larger systems, we will consider extending the theoretical results of [15, in order to use under- and over-approximations of the automaton $\mathcal{F}$, and refine these by counter-examples.

\section{References}

1. Abdellatif, T., Combaz, J., Sifakis, J.: Model-based implementation of real-time applications. In: EMSOFT 2010, pp. 229-238. ACM (2010)

2. Altisen, K., Tripakis, S.: Implementation of Timed Automata: An Issue of Semantics or Modeling? In: Pettersson, P., Yi, W. (eds.) FORMATS 2005. LNCS, vol. 3829, pp. 273-288. Springer, Heidelberg (2005)

3. Alur, R., Dill, D.L.: A theory of timed automata. Theoretical Computer Science 126(2), 183-235 (1994)

4. André, É., Fribourg, L., Kühne, U., Soulat, R.: IMITATOR 2.5: A tool for analyzing robustness in scheduling problems. In: Giannakopoulou, D., Méry, D. (eds.) FM 2012. LNCS, vol. 7436, pp. 33-36. Springer, Heidelberg (2012)

5. Bouyer, P., Markey, N., Sankur, O.: Robust Reachability in Timed Automata: A Game-Based Approach. In: Czumaj, A., Mehlhorn, K., Pitts, A., Wattenhofer, R. (eds.) ICALP 2012, Part II. LNCS, vol. 7392, pp. 128-140. Springer, Heidelberg (2012)

6. Bouyer, P., Markey, N., Sankur, O.: Robust model-checking of timed automata via pumping in channel machines. In: Fahrenberg, U., Tripakis, S. (eds.) FORMATS 2011. LNCS, vol. 6919, pp. 97-112. Springer, Heidelberg (2011)

7. Bozga, M., Daws, C., Maler, O., Olivero, A., Tripakis, S., Yovine, S.: Kronos: A model-checking tool for real-time systems. In: Vardi, M.Y. (ed.) CAV 1998. LNCS, vol. 1427, pp. 546-550. Springer, Heidelberg (1998) 
8. Cassez, F., Henzinger, T.A., Raskin, J.-F.: A comparison of control problems for timed and hybrid systems. In: Tomlin, C.J., Greenstreet, M.R. (eds.) HSCC 2002. LNCS, vol. 2289, pp. 134-148. Springer, Heidelberg (2002)

9. Daws, C., Yovine, S.: Two examples of verification of multirate timed automata with kronos. In: RTSS 1995, pp. 66-75. IEEE Computer Society Press (1995)

10. De Wulf, M., Doyen, L., Markey, N., Raskin, J.-F.: Robust safety of timed automata. FMSD 33(1-3), 45-84 (2008)

11. De Wulf, M., Doyen, L., Raskin, J.-F.: Almost ASAP semantics: From timed models to timed implementations. Formal Aspects of Computing 17(3), 319-341 (2005)

12. Henzinger, T.A., Sifakis, J.: The Embedded Systems Design Challenge. In: Misra, J., Nipkow, T., Sekerinski, E. (eds.) FM 2006. LNCS, vol. 4085, pp. 1-15. Springer, Heidelberg (2006)

13. Kordy, P., Langerak, R., Polderman, J.W.: Re-verification of a lip synchronization protocol using robust reachability. In: FMA, pp. 49-62 (2009)

14. Puri, A.: Dynamical properties of timed automata. Discrete Event Dynamic Systems 10(1-2), 87-113 (2000)

15. Sankur, O., Bouyer, P., Markey, N.: Shrinking timed automata. In: FSTTCS 2011, Leibniz-Zentrum für Informatik. LIPIcs, vol. 13, pp. 90-102 (2011)

16. Tripakis, S., Yovine, S.: Analysis of timed systems using time-abstracting bisimulations. Form. Methods Syst. Des. 18(1), 25-68 (2001) 